\title{
Time Delay Predictions in a Modified Gravity Theory
}

\author{
J. W. Moffat \\ Perimeter Institute for Theoretical Physics, Waterloo, Ontario, N2L 2Y5, Canada \\ Department of Physics, University of Waterloo, Waterloo, Ontario N2L 3G1, Canada \\ E-mail: john.moffat@utoronto.ca
}

\begin{abstract}
The time delay effect for planets and spacecraft is obtained from a fully relativistic modified gravity theory including a fifth force skew symmetric field by fitting to the Pioneer 10/11 anomalous acceleration data. A possible detection of the predicted time delay corrections to general relativity for the outer planets and future spacecraft missions is considered. The time delay correction to GR predicted by the modified gravity is consistent with the observational limit of the Doppler tracking measurement reported by the Cassini spacecraft on its way to Saturn, and the correction increases to a value that could be measured for a spacecraft approaching Neptune and Pluto
\end{abstract}

\section{Introduction}

In previous work [1], we have provided a possible gravitational explanation for the Pioneer 10/11 anomalous acceleration, based on the scalar-tensor-vector gravity (STVG) theory [2]. In the following we will obtain predictions for the corrections to the GR (general relativity) Shapiro time delay $[3,4,5,6,7]$, obtained from the fitted anomalous acceleration observed in the Pioneer 10/11 spacecraft data $[8,9,10,11,12]$. For our study of the solar system, we must account in our modified gravity theory for the variation of $G$ with respect to the radial distance $r$ from the center of the Sun. Since we do not possess rigorous solutions of the field equations, we use a phenomenological parameterization of the varying parameters $\alpha(r)$ and $\lambda(r)$ to obtain fits to the anomalous Pioneer acceleration data that are consistent with the solar system and fifth force experimental bounds. Thus, with the variation of $G$ with distance from the Sun, we can make predictions for other observational tests in the solar system. In the following, we shall calculate the corrections to the GR time delay effect for planets and spacecraft probes obtained from the STVG modified gravity theory.

\section{Pioneer Anomalous Acceleration}

We assume that gravity is the cause of the Pioneer 10/11 anomaly due to the difference between the running $G(r)$ and the bare value, $G_{0} \sim G_{N}$, where $G_{N}$ denotes the 
Newtonian gravitational constant. The Pioneer anomalous acceleration directed towards the center of the Sun is given by $[1,2]$ :

$$
a_{P}=-\frac{\delta G(r) M_{\odot}}{r^{2}}
$$

where

$$
\delta G(r)=G_{N} \alpha(r)\left[1-\exp (-r / \lambda(r))\left(1+\frac{r}{\lambda(r)}\right)\right] .
$$

We will use the following parametric representations of the "running" of $\alpha(r)$ and $\lambda(r)[1]:$

$$
\begin{aligned}
& \alpha(r)=\alpha_{\infty}(1-\exp (-r / \bar{r}))^{b / 2} \\
& \lambda(r)=\lambda_{\infty}(1-\exp (-r / \bar{r}))^{-b} .
\end{aligned}
$$

Here, $\bar{r}$ is a non-running distance scale parameter and $b$ is a constant $\neq$.

A best fit to the acceleration data extracted from Ref. [11] has been obtained using a nonlinear least-squares fitting routine including estimated errors from the Doppler shift observations [9]. The best fit parameters are [1]:

$$
\begin{aligned}
& \alpha_{\infty}=(1.00 \pm 0.02) \times 10^{-3}, \\
& \lambda_{\infty}=47 \pm 1 \mathrm{AU}, \\
& \bar{r}=4.6 \pm 0.2 \mathrm{AU}, \\
& b \quad=4.0 .
\end{aligned}
$$

The small uncertainties in the best fit parameters are due to the remarkably low variance

of residuals corresponding to a reduced $\chi^{2}$ per degree of freedom of 0.42 signalling a good fit.

\section{Time Delay in Modified Gravity}

Our scalar-tensor-vector gravity (STVG) theory does not have an exact spherically symmetric static solution. However, we have shown that the solution will have the form of the Schwarzschild solution for large values of the radial coordinate $r$ [2]. In the derivation of the line element, we have neglected the "running" of $G$. The line element in isotropic coordinates is given by

$$
d s^{2}=\frac{\left(1-\frac{G M}{2 c^{2} r}\right)^{2}}{\left(1+\frac{G M}{2 c^{2} r}\right)^{2}} c^{2} d t^{2}-\left(1+\frac{G M}{2 c^{2} r}\right)^{4} d \sigma^{2},
$$

where

$$
d \sigma^{2}=d r^{2}+r^{2} d \theta^{2}+r^{2} \sin ^{2} \theta d \phi^{2} .
$$

$\ddagger$ Note that in ref. [1] the exponent $b$ in Equation (12) should be $-b$. 
Expanding in powers of $G M / r$, we obtain in Cartesian coordinates

$$
d s^{2}=\left[1-\frac{2 G M}{c^{2} r}+2\left(\frac{G M}{c^{2} r}\right)^{2}\right] c^{2} d t^{2}-\left(1+\frac{2 G M}{c^{2} r}\right)\left(d x^{2}+d y^{2}+d z^{2}\right),
$$

where $r=\left(x^{2}+y^{2}+z^{2}\right)^{1 / 2}, \theta=\arctan \left[z /\left(x^{2}+y^{2}\right)^{1 / 2}\right]$ and $\phi=\arctan (y / x)$. The Sun is taken to be at the origin of coordinates and Earth and the spacecraft (planet) lie in the $z=0$ plane and the transmission null ray lies along the $\mathrm{x}$ direction. For a null ray

$$
d s^{2}=g_{00} c^{2} d t^{2}+g_{11} d x^{2}=0 .
$$

We now replace $G$ in (8) by $G_{N}+\delta G$ where $\delta G$ is given by (2) and obtain the correction to the GR round-trip time delay:

$$
\delta \tau=\left(\frac{4 M_{\odot}}{c^{3}}\right) \int_{-r_{\oplus}}^{r_{p}} d x\left[\frac{\delta G\left(\left(x^{2}+r_{0}^{2}\right)^{1 / 2}\right)}{\left(x^{2}+r_{0}^{2}\right)^{1 / 2}}\right],
$$

where $r_{\oplus}$ and $r_{p}$ are the distances of the Sun from Earth and the spacecraft (planet), respectively, and $r_{0}=y=$ const. The correction $\delta \tau$ in (10) is obtained by performing a numerical integration. The excess time delay for a round-trip delay is given in GR by

$$
(\Delta \tau)_{G R}=\frac{4 G_{N} M_{\odot}}{c^{3}} \ln \left(\frac{4 r_{p} r_{\oplus}}{r_{0}^{2}}\right) .
$$

The excess delay is calculated for a signal that grazes the limb of the Sun, $r_{0} \sim R_{\odot}$, and it is a maximum when the spacecraft (planet) is at superior conjunction.

In ref. [1], the variation of $\delta G / G_{N}$ arising from Equation (2) for the parametric values of $\alpha(r)$ and $\lambda(r)$ of Equation (3) and Equation (4), respectively, was obtained using the best fit values for the parameters given in Equation (5). The behavior of $G(r) / G_{N}$ is closely constrained to unity over the inner planets until beyond the orbit of Saturn $(r \gtrsim 10 \mathrm{AU})$ where the deviation of $G(r)$ from Newton's constant increases to an asymptotic value of $G_{\infty} / G_{N} \rightarrow 1.001$ over a distance of hundreds of AU.

In Table 1, we display the predicted values of the GR time delay $(\Delta \tau)_{\mathrm{GR}}$, the correction $\delta \tau$ obtained from STVG and the observational limit.

Table 1. Theoretical predictions for the corrections $\delta \tau$ to the GR time delays compared to the observational limits.

\begin{tabular}{lccc}
\hline $\mathrm{r}$ & $\begin{array}{c}\text { GR prediction } \\
(\mathrm{AU})\end{array}$ & $\begin{array}{c}\text { STVG prediction } \\
\delta \tau(\mu \mathrm{sec})\end{array}$ & $\begin{array}{c}\text { Observational limit } \\
(\Delta \tau)_{\mathrm{GR}}(\mu \mathrm{sec})\end{array}$ \\
\hline 1.52 (Mars) & 247 & $3.07 \times 10^{-12}$ & 0.5 \\
5.20 (Jupiter) & 271 & $3.10 \times 10^{-7}$ & - \\
8.43 (Cassini) & 262 & $8.71 \times 10^{-6}$ & $6.0 \times 10^{-3}$ \\
19.22 (Uranus) & 297 & $3.49 \times 10^{-4}$ & - \\
30.06 (Neptune) & 305 & $1.15 \times 10^{-3}$ & - \\
39.52 (Pluto) & 311 & $2.06 \times 10^{-3}$ & - \\
100 & 329 & $9.32 \times 10^{-3}$ & - \\
\hline
\end{tabular}

The Cassini spacecraft while on its way to Saturn reported the most accurate Doppler tracking measurement of the time delay effect [6]. The accuracy of the 
measurement was made possible by using both X-band (7175 MHz) and Ka-band (34316 $\mathrm{MHz}$ ) radar, thereby reducing significantly the dispersive effects of the solar corona. The 2002 superior conjunction of Cassini was favorable with the spacecraft at 8.43 AU from the Sun, and the distance of closest approach of the radar signals to the Sun was $r_{0} \sim 1.6 R_{\odot}$.

We see from the result for the correction to the GR time delay for the Cassini space probe that the prediction is well below the observational limit of $6.0 \times 10^{-3} \mu$ sec. The reported value for the measured post-Newtonian parameter $\gamma$ is $\gamma-1=(2.1 \pm 2.3) \times 10^{-5}$. Forthcoming new data for the anomalous acceleration will provide a more accurate determination of the parameters for $\alpha(r)$ and $\lambda(r)$ and the predicted values of $\delta \tau$.

A spacecraft orbiting Neptune and Pluto or on its way out of the solar system could hopefully report a sufficiently accurate Doppler tracking measurement of the time delay that could detect the correction to the GR prediction. At the positions of Neptune and Pluto and for a spacecraft at a distance from the Sun, $r_{0}=100 \mathrm{AU}$, the corrections $\delta \tau$ to the GR time delay are of the order of the observational limit of the Cassini Doppler tracking measurement, so Doppler measurements with comparable accuracy reported by a spacecraft approaching Neptune and Pluto and beyond the solar system could distinguish GR from the modified gravity theory prediction based on the Pioneer 10/11 data.

The Doppler measurements should be carried out at conjunction with the spacecraft (planet) to maximize the effects of the curvature of space at the distance of closest approach to the Sun. Moreover, Doppler measurements should be reported by the spacecraft (planet) at opposition to determine a baseline that yields the Newtonian time taken by a signal to reach the spacecraft (planet) and return to Earth. This latter timing will require using the JPL ephemeris including the modified gravity (STVG) to determine the corrected orbital parameters of the spacecraft (planet). Subtracting the baseline timing from the measurements at the distance of closest approach to the Sun will result in a residual correction $\delta t$ that can test the modified gravity theory. It should be noted that the calculation of $\delta t$ is not significantly sensitive to increasing values of $r_{0}$.

\section{Conclusions}

We have demonstrated in ref. [1] that the STVG theory can explain the Pioneer anomalous acceleration data and still be consistent with the accurate equivalence principle, lunar laser ranging and satellite data for the inner solar system as well as the outer solar system planets including Pluto at a distance of $r=39.52 \mathrm{AU}=5.91 \times 10^{12}$ meters. The ephemerides for the outer planets are not as well know as the ones for the inner planets due to their large distances from the Sun. The orbital data for Pluto only correspond to the planet having gone round $1 / 3$ of its orbit. It is important that the distance range parameter lies in the region $47 \mathrm{AU}<\lambda(r)<\infty$ for the best fit to the Pioneer acceleration data, for the range in the modified Yukawa correction to Newtonian 
gravity lies in a distance range beyond Pluto.

Perhaps, a future deep space probe can produce data that can check the predictions for the corrections to the time delays obtained from the Pioneer anomaly acceleration data and from our modified gravity theory. An analysis of anomalous acceleration data obtained from earlier Doppler shift data retrieval will clarify in better detail the apparent onset of the anomalous acceleration beyond the position of Saturn's orbit.

\section{Acknowledgments}

This work was supported by the Natural Sciences and Engineering Research Council of

Canada. I thank Joel Brownstein, Martin Green, Kenneth Nordtvedt, Viktor Toth and Slava Turyshev for helpful discussions.

\section{References}

[1] Brownstein J. R. and Moffat J W. 2006 Class. Quant. Grav. 233427 - 3436 (Preprint gr-qc/0511026)

[2] Moffat J. W. 2006 JCAP 0603004 (Preprint gr-qc/0506021)

[3] Shapiro I. I. 1964 Phys. Rev. Lett. 13, $789-791$

[4] Shapiro I. I. 1999 Rev. Mod. Phys. 71 S41-S53

[5] ReasenbergR. D., Shapiro I. I., MacNeil P.E., Goldstein R. B., BreidenthalJ.P., BrenkleJ.P., Cain D. L., Kaufman T. M., Komarek T.A., Zygielbaum A.I. 1979 Astrophys. J. 234, L219$\mathrm{L} 221$

[6] Bertotti B., Iess I., and Tortora P. 2003 Nature $425374-376$

[7] Will C. W. 2005 (Preprint gr-qc/0510072)

[8] Anderson J. D., Laing P. A., Lau E. L., Liu A. S., Nieto M. M. and Turyshev S. G. 1998 Phys. Rev. Lett. 81 2858-61 (Preprint gr-qc/9808081)

[9] Anderson J. D., Laing P. A., Lau E. L., Liu A. S., Nieto M. M. and Turyshev S. G. 2002 Phys. Rev. D 65082004 (Preprint gr-qc/0104064)

[10] Turyshev S. G., Nieto M. M. and Anderson J. D. 2005 (Preprint gr-qc/0510081)

[11] Nieto M. M. and Anderson J. D. 2005 Class. Quant. Grav. 225343 - 5354 (Preprint gr-qc/0507052)

[12] Turyshev S. G., Shao M. and Nordtvedt K. L. 2004 Int. J. Mod. Phys. D 13 2035-64 (Preprint gr-qc/0410044) 